MPP-2010-12

\title{
Triggering collective oscillations by three-flavor effects
}

\author{
Basudeb Dasgupta, ${ }^{1}$ Georg G. Raffelt, ${ }^{1}$ and Irene Tamborra ${ }^{1,2,3}$ \\ ${ }^{1}$ Max-Planck-Institut für Physik (Werner-Heisenberg-Institut), Föhringer Ring 6, 80805 München, Germany \\ ${ }^{2}$ Dipartimento Interateneo di Fisica "Michelangelo Merlin," Via Amendola 173, 70126 Bari, Italy \\ ${ }^{3}$ Istituto Nazionale di Fisica Nucleare, Sezione di Bari, Via Orabona 4, 70126 Bari, Italy
}

\begin{abstract}
Collective flavor transformations in supernovae, caused by neutrino-neutrino interactions, are essentially a two-flavor phenomenon driven by the atmospheric mass difference and the small mixing angle $\theta_{13}$. In the two-flavor approximation, the initial evolution depends logarithmically on $\theta_{13}$ and the system remains trapped in an unstable fixed point for $\theta_{13}=0$. However, any effect breaking exact $\nu_{\mu}-\nu_{\tau}$ equivalence triggers the conversion. Such three-flavor perturbations include radiative corrections to weak interactions, small differences between the $\nu_{\mu}$ and $\nu_{\tau}$ fluxes, or non-standard interactions. Therefore, extremely small values of $\theta_{13}$ are in practice equivalent, the fate of the system depending only on the neutrino spectra and their mass ordering.
\end{abstract}

PACS numbers: 14.60.Pq, 97.60.Bw

\section{INTRODUCTION}

Dense neutrino ensambles exhibit collective flavor transformations [1 $\left[\frac{6}{6}\right]$. For supernova (SN) neutrinos, these effects imprint intriguing features on the processed spectrum such as spectral splits [7-19]. Collective oscillations originate from a characteristic instability in flavor space of the interacting dense neutrino system that partly swaps its flavor content to minimize its energy. Assuming the primary $\nu_{e}$ and $\bar{\nu}_{e}$ fluxes exceed those of the other flavors, the dominant effect arises for inverted neutrino mass hierarchy. It is driven by the atmospheric mass difference and the mixing angle $\theta_{13}$ that is known to be small and could vanish entirely.

The transformation arising from an instability implies that the processed spectrum is independent of the mixing angle as long as it is small (collective transformations in the presence of matter are suppressed for maximal mixing because one projects on the interaction direction, but this $\cos 2 \theta$ effect is irrelevant if $\theta \ll 1[20,22])$. In a two-flavor treatment, $\theta$ enters only as a trigger to the subsequent evolution, so in the SN context a very small $\theta$ shifts logarithmically the onset radius for collective transformations [8, 9]. In numerical studies, choosing $\theta$ as small as allowed by the machine precision barely impacts the processed spectrum, although for $\theta=0$ the system remains stuck in the unstable fixed-point solution defined by the initial conditions.

Such a situation looks unphysical - it does not seem plausible that, at least in principle, one can distinguish between $\theta$ being exactly zero and some arbitrarily small but non-zero value. One may speculate, for example, that quantum fluctuations could trigger the transformation even for $\theta=0$ [8], noting that collective transformations actually preserve flavor lepton number.

The purpose of our paper is to show that in real life we do not need to worry about such subtleties. If $\theta_{13}$ is sufficiently small, three-flavor effects 20, 23 27] trigger the instability and the logarithmic $\theta_{13}$ dependence saturates at a small but non-zero value.
Why are collective SN neutrino transformations an effective two-flavor phenomenon anyway? In the outer layers the temperature is too low to support thermal $\mu$ or $\tau$ populations, obviating the possibility to distinguish between $\nu_{\mu}$ and $\nu_{\tau}$ by charged-current reactions. Ignoring radiative corrections, these flavors are exactly equivalent, allowing us to define new flavors $\nu_{x}$ and $\nu_{y}$ such that effectively $\theta_{23}=0$. If in addition $\theta_{13}=0$, one of the new states, say $\nu_{y}$, becomes equivalent to $\nu_{3}$, decoupling entirely from the other flavors: We are left with a twoflavor system consisting of $\nu_{e}$ and $\nu_{x}$, governed by $\theta_{12}$ and the solar mass difference $\delta m^{2}$.

This system can show collective transformations. However, the solar mass hierarchy is normal, suppressing the dominant transformation effect if the primary fluxes show the usual excess of $\nu_{e}$ and $\bar{\nu}_{e}$. Moreover, the collective oscillation region is at larger radii because the solar mass difference is small, so multiple-split effects are more easily suppressed by adiabaticity violation [12, 13]. However, collective oscillations driven by the solar mass difference do modify the spectra in some scenarios 21].

Once we allow for a small but non-vanishing $\theta_{13}$, collective $\nu_{e} \leftrightarrow \nu_{y}$ transformations become possible that are driven by the atmospheric mass difference $\Delta m^{2}$ and occur in the usual region of large neutrino flux densities. Our main point is that for $\theta_{13}=0$ these transformations are triggered by small perturbations of the exact $\nu_{\mu}-\nu_{\tau}$ equivalence because $\nu_{e}$ and $\nu_{x}$ then no longer form an exact two-flavor system. Such perturbations include radiative corrections to the $\nu_{\mu}$ and $\nu_{\tau}$ matter effect [28, 29], or small $\nu_{\mu}-\nu_{\tau}$ flux differences. The latter can be caused by the presence of muons in deeper layers of the $\mathrm{SN}$ core and by radiative corrections to the interaction rates, modifying the relative $\nu_{\mu}$ and $\nu_{\tau}$ opacities. Non-standard interactions can also break the $\nu_{\mu}-\nu_{\tau}$ symmetry [27], a possibility that we will not pursue here.

We begin in Sec. III with a brief discussion of the equations of motion. In Sec. III we prove that for $\theta_{13}=0$ and for exact $\nu_{\mu}-\nu_{\tau}$ equivalence, collective oscillations driven by the atmospheric mass difference are not possible, justifying the usual two-flavor treat- 
ment. In Sec. IV we study concrete departures from $\nu_{\mu}-\nu_{\tau}$ equivalence in the limit $\theta_{13}=0$ and show that collective transformations are triggered by these effects. In schematic models we compare them with an equivalent $\theta_{13}$ that would trigger collective transformations at the same onset radius. In Sec. $\mathrm{V}$ we consider a realistic SN and study the competition between a small $\theta_{13}$ and a small $\nu_{\mu}-\nu_{\tau}$ flux difference. We conclude with a brief summary in Sec. VI.

\section{EQUATIONS OF MOTION}

\section{A. Matrix form}

For our conceptual discussion it is sufficient to consider the simplest three-flavor system showing collective transformations. We take the neutrino ensemble to be homogeneous and isotropic, study its time evolution, and describe mixed neutrinos by matrices of densities $\varrho_{E}$ for each energy mode $E$. We use an overbar to represent the corresponding quantities for antineutrinos. Diagonal entries are the usual occupation numbers, whereas offdiagonal entries encode phase information. The equations of motion (EoM) are

$$
\mathrm{i} \dot{\varrho}_{E}=\left[\mathrm{H}_{E}, \varrho_{E}\right] \text { and i } \dot{\bar{\varrho}}_{E}=\left[\overline{\mathrm{H}}_{E}, \bar{\varrho}_{E}\right] \text {. }
$$

The Hamiltonian $3 \times 3$ matrix is made up of the vacuum, matter, and neutrino-neutrino terms

$$
\mathrm{H}_{E}=\mathrm{H}_{E}^{\mathrm{vac}}+\mathrm{H}^{\lambda}+\mathrm{H}^{\nu \nu} \text {. }
$$

Here $\mathrm{H}_{E}^{\mathrm{vac}}=\mathrm{UM}^{2} \mathrm{U}^{\dagger} / 2 E$, with $\mathrm{U}=\mathrm{R}_{23} \mathrm{R}_{13} \mathrm{R}_{12}$ the neutrino mixing matrix and $\mathrm{M}=\operatorname{diag}\left(m_{1}, m_{2}, m_{3}\right)$ the mass matrix. We use the standard notation of $\mathrm{R}_{i j}$ as the rotation matrix between the $i$ and $j$ mass eigenstates, with argument $\theta_{i j}$. For antineutrinos, the vacuum Hamiltonian picks up a relative minus $\operatorname{sign}\left(\bar{H}_{E}^{\mathrm{vac}}=-\mathrm{H}_{E}^{\mathrm{vac}}\right)$, whereas all other pieces remain identical. For oscillation studies, we may neglect terms proportional to the identity and may write in the mass basis

$$
\mathrm{H}_{E}^{\mathrm{vac}}=\frac{1}{2 E} \operatorname{diag}\left(-\frac{\delta m^{2}}{2},+\frac{\delta m^{2}}{2}, \Delta m^{2}\right) .
$$

The solar mass-squared difference $\delta m^{2}>0$, whereas the atmospheric one $\Delta m^{2}<0$ for inverted mass hierarchy (IH) and $\Delta m^{2}>0$ for normal hierarchy (NH).

The matter term, due to neutrino interactions with the charged leptons, is in the flavor basis

$$
\mathrm{H}^{\lambda}=\sqrt{2} G_{\mathrm{F}} \operatorname{diag}\left(N_{e}, N_{\mu}, N_{\tau}\right)+\mathcal{O}\left(G_{\mathrm{F}}^{2}\right),
$$

where $N_{e}$ is the net electron density (electrons minus positrons) and similarly for the other leptons. The second-order term is due to radiative corrections and can be non-negligible at high densities [28]. The contribution associated to $\nu$ - $\nu$ interactions is

$$
\mathrm{H}^{\nu \nu}=\sqrt{2} G_{\mathrm{F}} \int \mathrm{d} E\left(\varrho_{E}-\bar{\varrho}_{E}\right)+\mathcal{O}\left(G_{\mathrm{F}}^{2}\right) .
$$

Multi-angle effects are ignored in our isotropic system. Radiative corrections can be important in dense neutrino gases at the second order [29].

\section{B. New interaction basis $e^{-x}-\boldsymbol{y}$}

Since we are concerned with a system where the $\nu_{\mu}$ and $\nu_{\tau}$ flavors are exactly or approximately equivalent, it is more useful to introduce new flavors $x$ and $y$ that simplify the mixing matrix 24]

$$
\left(\begin{array}{l}
\nu_{e} \\
\nu_{x} \\
\nu_{y}
\end{array}\right)=\mathrm{R}_{23}^{\dagger}\left(\begin{array}{c}
\nu_{e} \\
\nu_{\mu} \\
\nu_{\tau}
\end{array}\right)
$$

Here $\mathrm{R}_{23}^{\dagger}$ "unmixes" $\nu_{\mu}$ and $\nu_{\tau}$ with the angle $\theta_{23}$. For $\theta_{13}=0, \nu_{y}$ is the mass eigenstate $\nu_{3}$. Henceforth the interaction basis is understood to be the $e-x-y$-basis.

This basis is useful because it explicitly removes $\theta_{23}$ from the formalism, if the Hamiltonian and the initial conditions do not distinguish $\nu_{\mu}$ and $\nu_{\tau}$. Naturally, the evolution of $\nu_{e}$ and $\bar{\nu}_{e}$ is independent of $\theta_{23}$ in this approximation.

\section{Expansion in Gell-Mann matrices}

The commutator structure of the equations of motion ensures that the trace of $\varrho_{E}$ is conserved, so we may re-define them to be traceless by subtracting a term proportional to the identity matrix I. The traceless part can be expanded in Gell-Mann matrices $\Lambda_{i}$ with the expansion coefficients forming an 8 -vector $\mathbf{X}$. Thus one can project any matrix $\mathrm{X}$ as

$$
\mathrm{X}=\operatorname{tr}(\mathrm{X})\left(\frac{\mathrm{I}}{3}+\mathrm{X} \cdot \frac{\boldsymbol{\Lambda}}{2}\right)
$$

where $\boldsymbol{\Lambda}$ is an 8 -vector of $\Lambda$ matrices. We normalize as $|\mathbf{X}|=2 / \sqrt{3}$, corresponding to the conventions $\Lambda_{i}=\Lambda_{i}^{\dagger}$, $\operatorname{tr}\left(\Lambda_{i}\right)=0, \operatorname{tr}\left(\Lambda_{i} \Lambda_{j}\right)=2 \delta_{i j}$, and $\left[\Lambda_{i}, \Lambda_{j}\right]=2 i f_{i j k} \Lambda_{k}$. Here $f_{i j k}$ are the $\mathrm{SU}(3)$ structure constants, where

$$
\begin{aligned}
& f_{123}=1 \\
& f_{147}=f_{246}=f_{257}=f_{345}=-f_{156}=-f_{367}=1 / 2 \\
& f_{458}=f_{678}=\sqrt{3} / 2
\end{aligned}
$$

are the non-vanishing values.

The neutrino matrices of density can now be decomposed, as in Eq. (7), in terms of an 8-dimensional polarization vector

$$
\varrho_{E}=n_{E}\left(\frac{\mathrm{I}}{3}+\mathbf{P}_{E} \cdot \frac{\boldsymbol{\Lambda}}{2}\right) .
$$

$n_{E}$ is the total neutrino density per unit energy interval. Analogous expressions pertain to antineutrinos. 
The different parts of the Hamiltonian can also be similarly decomposed. The vacuum Hamiltonian is

$$
\mathrm{H}_{E}^{\mathrm{vac}}=\omega_{E}\left(\frac{\mathrm{I}}{3}+\mathbf{B} \cdot \frac{\boldsymbol{\Lambda}}{2}\right),
$$

where $\omega_{E}=\Delta m^{2} /(2 E)$. The "magnetic field" is

$$
\mathbf{B}=\left(\begin{array}{c}
0 \\
0 \\
s_{13}^{2} \\
S_{13} \\
0 \\
0 \\
0 \\
-\frac{1}{2 \sqrt{3}}\left(1+3 C_{13}\right)
\end{array}\right)+\epsilon_{\omega}\left(\begin{array}{c}
c_{13} S_{12} \\
0 \\
-\frac{1}{4} C_{12}\left(3+C_{13}\right) \\
\frac{1}{2} C_{12} S_{13} \\
0 \\
-s_{13} S_{12} \\
0 \\
\frac{\sqrt{3}}{2} C_{12} s_{13}^{2}
\end{array}\right),
$$

with $\epsilon_{\omega}=\delta m^{2} / \Delta m^{2}$. Moreover, we use $s_{i j}=\sin \theta_{i j}$, $c_{i j}=\cos \theta_{i j}, S_{i j}=\sin 2 \theta_{i j}$, and $C_{i j}=\cos 2 \theta_{i j}$.

Ignoring a term proportional to identity, the matter term can be written as

$$
\mathrm{H}^{\lambda}=\lambda \operatorname{diag}\left(1,0, \epsilon_{\lambda}\right)=\lambda\left(\frac{\mathrm{I}}{3}+\mathbf{L} \cdot \frac{\boldsymbol{\Lambda}}{2}\right) .
$$

Here $\lambda=\sqrt{2} G_{\mathrm{F}} N_{e}$ is the effective MSW potential. For later reference we have included $\epsilon_{\lambda} \ll 1$, encoding radiative corrections or small $\nu_{\mu}-\nu_{\tau}$ flux differences. The leptonic "magnetic field" is

$$
\mathbf{L}=\left(\begin{array}{c}
0 \\
0 \\
1 \\
0 \\
0 \\
0 \\
0 \\
\frac{1}{\sqrt{3}}
\end{array}\right)+\epsilon_{\lambda}\left(\begin{array}{c}
0 \\
0 \\
-s_{23}^{2} \\
0 \\
0 \\
-S_{23} \\
0 \\
-\frac{1}{2 \sqrt{3}}\left(1+3 C_{23}\right)
\end{array}\right)
$$

The $\nu$ - $\nu$ interaction term finally is

$$
\mathrm{H}^{\nu \nu}=\mu\left(\frac{\mathrm{I}}{3}+\mathbf{D} \cdot \frac{\boldsymbol{\Lambda}}{2}\right),
$$

where the effective neutrino-neutrino interaction energy is $\mu=\sqrt{2} G_{\mathrm{F}}(N+\bar{N})$. Here $N=N_{\nu_{e}}+N_{\nu_{\mu}}+N_{\nu_{\tau}}$ is the overall neutrino density, and $\bar{N}$ for antineutrinos. The collective vector $\mathbf{D}$ is explicitly

$$
\mathbf{D}=\int \mathrm{d} E \frac{n_{E} \mathbf{P}_{E}-\bar{n}_{E} \overline{\mathbf{P}}_{E}}{N+\bar{N}} .
$$

The EoM are then

$$
\begin{aligned}
& \dot{\mathbf{P}}_{E}=\left(+\omega_{E} \mathbf{B}+\lambda \mathbf{L}+\mu \mathbf{D}\right) \times \mathbf{P}_{E} \\
& \dot{\overline{\mathbf{P}}}_{E}=\left(-\omega_{E} \mathbf{B}+\lambda \mathbf{L}+\mu \mathbf{D}\right) \times \overline{\mathbf{P}}_{E} .
\end{aligned}
$$

The 8-dimensional vector product is defined as $(\mathbf{a} \times \mathbf{b})_{i}=$ $f_{i j k} a_{j} b_{k}$. In this form, the problem resembles a set of polarization vectors $\mathbf{P}_{E}$ precessing under the influence of the combined magnetic fields $\mathbf{B}, \mathbf{L}$, and the mean field $\mathbf{D}$ due to all polarization vectors.

\section{EXACT $\nu_{\mu}-\nu_{\tau}$ EQUIVALENCE}

In the approximation that nothing distinguishes between the $\nu_{\mu}$ and $\nu_{\tau}$ flavor, 2-3 mixing is physically irrelevant and we expect that oscillations reduce to a two-flavor problem. In fact, for $\theta_{13}=0$, no collective effects driven by the atmospheric mass difference occur.

To prove this point we study a simplified system consisting of two Bloch vectors, representing equal numbers of neutrinos and antineutrinos, with the single vacuum oscillation frequency $\omega$. For $\theta_{13}=0$ the magnetic field simplifies to

$$
\mathbf{B}=\frac{2}{\sqrt{3}} \mathbf{e}_{8}+\epsilon_{\omega}\left(S_{12} \mathbf{e}_{1}-C_{12} \mathbf{e}_{3}\right),
$$

where $\mathbf{e}_{i}$ are unit vectors in the 8-dimensional flavor space. Assuming exact $\nu_{\mu}-\nu_{\tau}$ equivalence implies that $\epsilon_{\lambda}=0$, and therefore

$$
\mathbf{L}=\mathbf{e}_{3}+\frac{1}{\sqrt{3}} \mathbf{e}_{8} .
$$

Likewise, if the initial $\nu_{\mu}$ and $\nu_{\tau}$ densities are equal, the initial polarization vectors $\mathbf{P}=\overline{\mathbf{P}}$ are proportional to the same linear combination of $\mathbf{e}_{3}$ and $\mathbf{e}_{8}$.

The static vectors $\mathbf{B}$ and $\mathbf{L}$ have components in the 1 , 3 , and 8 directions, whereas the only dynamical component of $\mathbf{H}$, the self-term $\mathbf{D}$, develops an $\mathbf{e}_{2}$ component. The EoM of the $\mathbf{D}$ vector derives from the difference of Eqs. (16) and (17)

$$
\dot{\mathbf{D}}=-\epsilon_{\omega} \omega\left[S_{12}\left(P_{3}+\bar{P}_{3}\right)+C_{12}\left(P_{1}+\bar{P}_{1}\right)\right] \mathbf{e}_{2} .
$$

In other words, the vector $\mathbf{H}=\omega \mathbf{B}+\lambda \mathbf{L}+\mu \mathbf{D}$ has only components in the $1,2,3$ and 8 direction and thus can not mix $\nu_{e}$ and $\nu_{y}$.

The same conclusion is reached if we consider the EoM in matrix form. The part consisting of the $e$ and $x$ flavor and the $y$ flavor form separate block matrices both for the Hamiltonian matrix and the matrices of densities. In the $e-x-y$ basis and with $\theta_{13}=0$, the third mass eigenstate $\nu_{3}$ is not admixed to the $\nu_{e}$ and $\nu_{x}$ flavors.

\section{BROKEN $\nu_{\mu}-\nu_{\tau}$ EQUIVALENCE}

Even in the absence of thermal $\mu$ or $\tau$ populations the exact $\nu_{\mu}-\nu_{\tau}$ equivalence is broken by several subleading effects that distinguish between these flavors. In this case, the $\nu_{3}$ flavor does not fully decouple from the $\nu_{e}-\nu_{x}$ system and collective transitions driven by the atmospheric mass difference are inevitably triggered.

The first is provided by radiative corrections to the neutrino matter effect where charged leptons appear in the loop. Even in the absence of ordinary matter, similar radiative corrections arise for neutrino-neutrino interactions, although the detailed structure of the EoM becomes more complicated [29]. Since collective effects 
require a large density of neutrinos, radiative corrections and thus the breaking of $\nu_{\mu}-\nu_{\tau}$ equivalence are unavoidable. Finally we note that differences in the initial $\nu_{\mu}$ and $\nu_{\tau}$ fluxes also provides the required instability.

\section{A. Radiative corrections to $\nu_{\tau}$ matter effect}

The presence of matter (i.e. $\lambda \neq 0$ ) has a similar effect as decreasing the effective mixing angle, although in detail the dynamics is more complicated. In a frame rotating around $\mathbf{L}$ there is a fast-rotating transverse $B$ field that disturbs the system and triggers the evolution [8]. However, if matter effects distinguish $\nu_{\mu}$ and $\nu_{\tau}$, they can play a more important role in trigerring collective oscillations, particularly for a small mixing angle. The largest correction is for $\nu_{\tau}$ where a background of ordinary matter with baryon density $N_{B}$ has the same refractive effect on $\nu_{\tau}$ and $\bar{\nu}_{\tau}$ that would be provided by a density of real $\tau$ leptons $\left(N_{\tau}^{\text {eff }}=2.6 \times 10^{-5} N_{B}\right)[28]$. This subleading correction is parametrized as $\epsilon_{\lambda}$ to the usual matter effect $\lambda$ in Eq. (13).

Off-diagonal terms in the Hamiltonian generated by $\epsilon_{\lambda}$ will mix $\nu_{e}$ and $\nu_{y}$. In the limit $\theta_{13} \rightarrow 0$, when $\nu_{3}$ would otherwise have decoupled, these terms play a role similar to $\theta_{13}$, and recouple $\nu_{3}$ to $\nu_{e}$. We can estimate the effective $\theta_{13}$ generated by these sub-leading matter effects by diagonalizing the Hamiltonian instantaneously in matter as

$$
\mathrm{H}_{E}^{\mathrm{vac}}+\mathrm{H}^{\lambda}=\frac{\widetilde{U} \widetilde{M}^{2} \widetilde{U}^{\dagger}}{2 E}
$$

where the $\widetilde{M}$ and $\widetilde{U}$ denote the mass and mixing matrix in matter. Using the standard parametrization, Eq. (21) can be solved for the parameters of $\widetilde{U}$ and $\widetilde{M}$. We find that the matter induced $1-3$ mixing is

$$
\tan 2 \widetilde{\theta}_{13}=\frac{2 \epsilon_{\lambda} \epsilon_{\omega} \lambda S_{12} S_{23}}{2 \omega+2 \epsilon_{\omega} \omega C_{12}+\left(\epsilon_{\lambda}-2\right) \lambda+\epsilon_{\lambda} \lambda C_{23}},
$$

where we ignore terms beyond the leading order in $\epsilon_{\lambda}$. This equation should be interpreted as providing the critical value of $\theta_{13}$ such that if $\theta_{13} \lesssim \widetilde{\theta}_{13}$, the role of $\nu_{\mu}-$ $\nu_{\tau}$ equivalence breaking is more important than $\theta_{13}$ itself, and the two-flavor approximation is not valid anymore.

To demonstrate this, we consider a toy model with one Bloch vector for neutrinos $\mathbf{P}$ and one for antineutrinos $\overline{\mathbf{P}}$ with equal length. In the two-flavor case this would be the simple flavor pendulum without intrinsic angular momentum. A nonvanishing mixing angle triggers an exponential growth of the misalignment between the force direction and the initial orientation. The time (or distance) after which an $\mathcal{O}(1)$ deviation from the initial orientation is achieved grows logarithmically with decreasing mixing angle. We define the radius at which there is a change of $1 \%$ in the $\nu_{e}$ flavor content as the onset radius. In Fig. 1, we show the onset radius for this system, and how it depends on $\theta_{13}$. We use

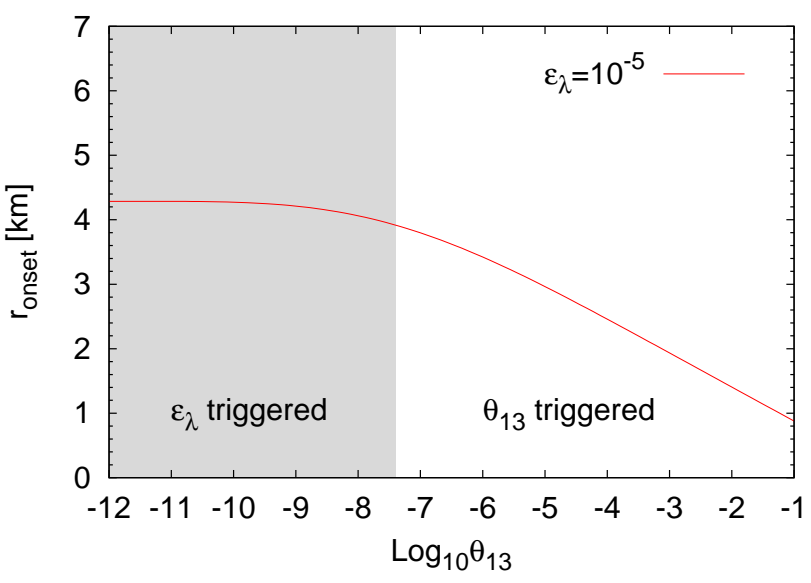

FIG. 1: Onset radius as a function of $\theta_{13}$ for the toy model described in the text. We assume a small difference between $\nu_{\mu}$ and $\nu_{\tau}$ refraction parameterized by $\epsilon_{\lambda}=10^{-5}$.

$\lambda=100 \mathrm{~km}^{-1}, \epsilon_{\lambda}=5 \times 10^{-5}, \mu=10 \mathrm{~km}^{-1}, \omega=1$ $\mathrm{km}^{-1}$, and the mixing angles $\theta_{12}=0.6, \theta_{23}=\pi / 4$. Using Eq. 22 for the chosen parameters, we expect that the matter induced mixing becomes important at $\theta_{13} \approx 10^{-7}$. This is in good agreement with what we find. The logarithmic increase of the onset radius stops below this critical mixing angle.

\section{B. Different primary $\nu_{\mu}$ and $\nu_{\tau}$ fluxes}

Another way to break the exact $\nu_{\mu}-\nu_{\tau}$ equivalence is through an initial flux difference. Although this effect is inevitable it has not been studied in detail. Deep in a SN core, the temperature is large enough to support a thermal muon population, slightly modifying the primary fluxes. Moreover, the same radiative effects that create a refractive difference between $\nu_{\mu}$ and $\nu_{\tau}$ also modify the scattering rates and the two flavors will have slightly different opacities and therefore different thermally driven fluxes. Obviously the discrete nature of particle emission and thermal fluctuations of the regions emitting the neutrinos would necessarily make the two spectra different.

As a toy example we again assume two equal Bloch vectors $\mathbf{P}$ and $\overline{\mathbf{P}}$. The difference between the initial densities of $\nu_{\mu}$ and $\nu_{\tau}$ is parameterized as

$$
\epsilon_{N}=\frac{N_{\nu_{\mu}}-N_{\nu_{\tau}}}{N_{\nu_{e}}}
$$

and the same for antineutrinos. Ignoring the matter effect, we have explicitly

$$
\dot{\mathbf{D}}=\omega \mathbf{B} \times(\mathbf{P}+\overline{\mathbf{P}}),
$$

which dynamically generates components of $\mathbf{D}$ along $\mathbf{e}_{5}$ and $\mathbf{e}_{7}$ even though initially $\mathbf{H}$ has only components in the $1,2,3$, and 8 directions. Therefore $\mathbf{H}$ develops a component along $\mathbf{e}_{5}$, leading to a mixing of $\nu_{e}$ and $\nu_{y}$. 


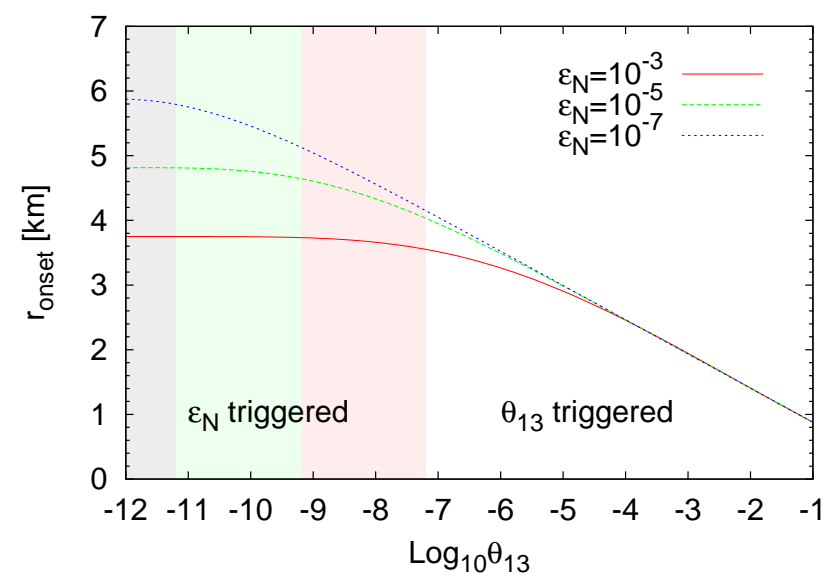

FIG. 2: Same as Fig. 1, now for equal $\nu_{\mu}$ and $\nu_{\tau}$ refraction, but for different fluxes as indicated by the values of $\epsilon_{N}$.

It is not straightforward to define an effective mixing angle in this case. The effect of the different fluxes for $\nu_{\mu}$ and $\nu_{\tau}$ is to provide terms proportional to $\epsilon_{N} \mu$ to the $\nu_{x^{-}}$ $\nu_{y}$ block in the Hamiltonian. These terms are themselves dynamical (time-dependent), and are communicated to the $\nu_{e}-\nu_{y}$ block by the mixing between $\nu_{e}$ and $\nu_{x}$. The effective mixing angle can be thought as being the initial misalignment of $\mathbf{P}$ from the Hamiltonian which is approximately proportional to $\epsilon_{N} /(\omega+\lambda)$. As this is a three-flavor effect, it must vanish when $\epsilon_{\omega} \rightarrow 0$. We therefore expect

$$
\widetilde{\theta}_{13} \sim \frac{\epsilon_{N} \epsilon_{\omega} \omega}{\omega+\lambda} .
$$

The logarithmic increase of $r_{\text {onset }}$ with decreasing $\theta_{13}$ saturates at $\theta_{13}$ approximately equal to the effective mixing $\widetilde{\theta}_{13}$ induced by unequal $\nu_{\mu}-\nu_{\tau}$ fluxes.

In Fig. 2, we plot $r_{\text {onset }}$ for this system as a function of $\theta_{13}$ for different values of $\epsilon_{N}$, illustrating this effect. We use the frequencies $(\omega, \mu, \lambda)=(1,10,100) \mathrm{km}^{-1}$, and the mixing angles $\sin ^{2} \theta_{12}=0.314$, and $\sin ^{2} \theta_{23}=0.5$. Using Eq. (25) for the chosen parameters, we expect the fluxasymmetry induced mixing to become important at $\theta_{13} \sim$ $\epsilon_{N} /\left(3 \times 10^{-3}\right)$. This is in good agreement with what we find. The logarithmic increase of the onset radius stops below the estimated value of the mixing angle.

\section{REALISTIC SUPERNOVA}

We finally consider a more realistic SN example in a single-angle treatment. The neutrinos are assumed to be emitted isotropically from the neutrinosphere at $R_{\nu}=$ $10 \mathrm{~km}$. We assume equal luminosities for all neutrino flavors, given by

$$
L=1.2 \times 10^{52} \mathrm{erg} / \mathrm{s},
$$

and thermal spectra with average energies $\left\langle E_{\nu_{e}}\right\rangle=10$, $\left\langle E_{\bar{\nu}_{e}}\right\rangle=15$, and $\left\langle E_{\bar{\nu}_{\mu, \tau}}\right\rangle=20 \mathrm{MeV}$. The electron density of the matter is the same as in [30] at $t=1 \mathrm{~s}$ after the bounce. For the neutrino mixing parameters we use

$$
\begin{aligned}
\Delta m^{2} & =2 \times 10^{-3} \mathrm{eV}^{2}, \\
\delta m^{2} & =8 \times 10^{-5} \mathrm{eV}^{2}, \\
\sin ^{2} \theta_{12} & =0.31, \\
\sin ^{2} \theta_{23} & =0.50 .
\end{aligned}
$$

With these assumptions, we have calculated the onset radius for collective transformations as a function of $\theta_{13}$, assuming a flux difference $\epsilon_{N}=10^{-5}$ and ignoring radiative corrections to the matter effect. Our results are shown in Fig. 3. For $\theta_{13} \lesssim 10^{-3}$, the onset radius is not sensitive to $\theta_{13}$, as expected from Eq. (25).

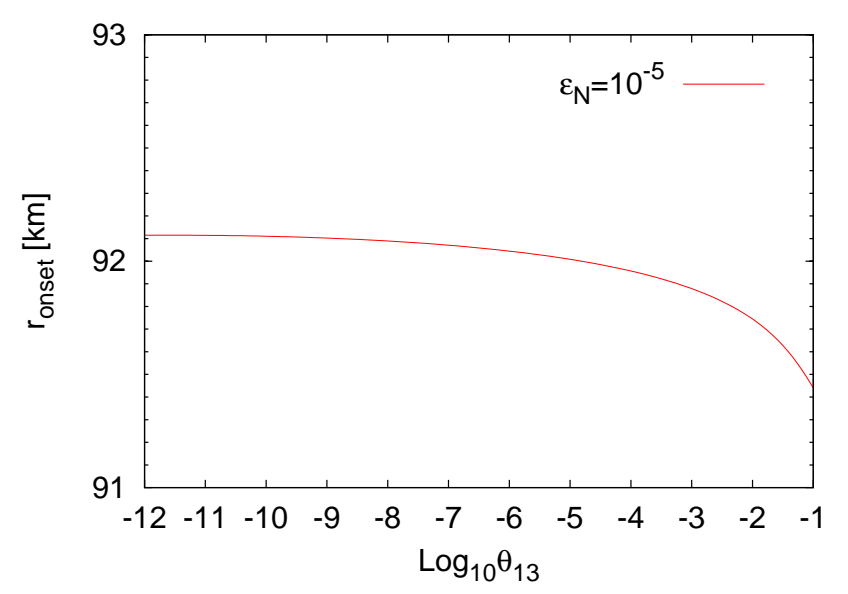

FIG. 3: Onset radius of collective oscillations for a realistic SN example, assuming $\epsilon_{N}=10^{-5}$.

In a realistic $\mathrm{SN}$, even when the $\nu_{\mu}-\nu_{\tau}$ equivalence is perfect, the onset radius depends only very weakly on $\theta_{13}$. The unavoidable breaking of this symmetry by radiative corrections and the the presence of charged muons in the deep SN core almost completely removes the $\theta_{13}$ dependence in the three-flavor context. Of course, MSW transitions caused by the ordinary matter effect depend on $\theta_{13}$ in the usual way.

\section{CONCLUSIONS}

Collective oscillations are an instability-driven phenomenon. The system transits from its initial unstable configuration to a stable one, triggered by the influence of a disturbance. Usually one thinks of this disturbance as being provided by the small offset between the relevant flavor and the propagation eigenstates, encoded into the mixing angle $\theta_{13}$. When this mixing angle is exactly vanishing, one would naively think that the oscillations do not take place.

However, one should recognize that a system sitting on an unstable fixed point is bound to be disturbed, unless there are symmetries that forbid all perturbations capable of providing an initial disturbance. In the neutrino 
oscillation context, this symmetry happens to be the $\mu-$ $\tau$ symmetry - which is explicitly broken. Consequently, collective oscillations are inevitable. This means that collective oscillations take place as usual even at $\theta_{13}=0$, once triggered by subleading effects.

Another fundamental point is that SN neutrino oscillations are not sensitive to arbitrarily small values of the mixing angle. The fantastic sensitivity to an arbitrarily small mixing angle, as it appears in two-flavor analyses, disappears when one takes into account other sub-leading corrections. As a result, strategies outlined in Refs. [15, 16] may be useful for determination of the mass hierarchy if the relevant signals are observed, but not for determination of a non-zero $\theta_{13}$ itself. On the other hand, in principle we could determine the neutrino mass hierarchy even if $\theta_{13}$ were exactly zero-which might end up being our only hope if $\theta_{13}$ is beyond the reach of laboratory-based oscillation experiments.

\section{Acknowledgements}

This work was partly supported by the Deutsche Forschungsgemeinschaft under grant TR-27 "Neutrinos and Beyond" and the Cluster of Excellence "Origin and Structure of the Universe" (Munich and Garching). The work of I.T. has been partly supported by the Italian MIUR and INFN through the "Astroparticle Physics" research project. Her stay in Munich has been partly supported by a junior fellowship awarded by the Italian Society of Physics (Borsa SIF "Antonio Stanghellini").
[1] J. T. Pantaleone, Phys. Lett. B 287, 128 (1992).

[2] G. Sigl and G. Raffelt, Nucl. Phys. B 406, 423 (1993).

[3] V. A. Kostelecký and S. Samuel, Phys. Rev. D 52, 621 (1995).

[4] S. Pastor, G. G. Raffelt and D. V. Semikoz, Phys. Rev. D 65, 053011 (2002).

[5] R. F. Sawyer, Phys. Rev. D 72, 045003 (2005).

[6] H. Duan, G. M. Fuller and Y.-Z. Qian, Phys. Rev. D 74, 123004 (2006).

[7] H. Duan, G. M. Fuller, J. Carlson and Y.-Z. Qian, Phys. Rev. D 74, 105014 (2006).

[8] S. Hannestad, G. G. Raffelt, G. Sigl and Y. Y. Y. Wong, Phys. Rev. D 74, 105010 (2006); Erratum ibid. 76, 029901 (2007).

[9] H. Duan, G. M. Fuller, J. Carlson and Y.-Z. Qian, Phys. Rev. D 75, 125005 (2007).

[10] G. G. Raffelt and A. Yu. Smirnov, Phys. Rev. D 76, 081301 (2007); Erratum ibid. 77, 029903 (2008).

[11] G. L. Fogli, E. Lisi, A. Marrone and A. Mirizzi, JCAP 0712, 010 (2007).

[12] G. L. Fogli, E. Lisi, A. Marrone, A. Mirizzi and I. Tamborra, Phys. Rev. D 78, 097301 (2008).

[13] B. Dasgupta, A. Dighe, G. G. Raffelt and A. Yu. Smirnov, Phys. Rev. Lett. 103, 051105 (2009).

[14] G. Fogli, E. Lisi, A. Marrone and I. Tamborra, JCAP 0910 (2009) 002.

[15] H. Duan, G. M. Fuller, J. Carlson and Y.-Z. Qian, Phys. Rev. Lett. 99, 241802 (2007).

[16] B. Dasgupta, A. Dighe and A. Mirizzi, Phys. Rev. Lett.
101, 171801 (2008).

[17] H. Duan, G. M. Fuller, J. Carlson and Y.-Z. Qian, Phys. Rev. Lett. 100, 021101 (2008).

[18] H. Duan, G. M. Fuller and Y.-Z. Qian, Phys. Rev. D 77, 085016 (2008).

[19] B. Dasgupta, A. Dighe, A. Mirizzi and G. G. Raffelt, Phys. Rev. D 77, 113007 (2008).

[20] A. Esteban-Pretel A. Mirizzi, S. Pastor, R. Tomàs, G. G. Raffelt, P. D. Serpico and G. Sigl, Phys. Rev. D 78, 085012 (2008).

[21] A. Friedland, arXiv:1001.0996 [hep-ph].

[22] A. Esteban-Pretel, PhD Thesis (University of Valencia, 2009), arXiv:0912.1616 [hep-ph].

[23] A. Esteban-Pretel, S. Pastor, R. Tomàs, G. G. Raffelt and G. Sigl, Phys. Rev. D 77, 065024 (2008).

[24] B. Dasgupta and A. Dighe, Phys. Rev. D 77, 113002 (2008).

[25] G. Fogli, E. Lisi, A. Marrone and I. Tamborra, JCAP 0904, 030 (2009).

[26] J. Gava and C. Volpe, Phys. Rev. D 78, 083007 (2008).

[27] M. Blennow, A. Mirizzi and P. D. Serpico, Phys. Rev. D 78, 113004 (2008).

[28] F. J. Botella, C. S. Lim and W. J. Marciano, Phys. Rev. D 35, 896 (1987).

[29] A. Mirizzi, S. Pozzorini, G. G. Raffelt and P. D. Serpico, JHEP 0910, 020 (2009).

[30] R. C. Schirato and G. M. Fuller, arXiv:astro-ph/0205390 